\title{
How Is Mass Spectrometry Tackling the COVID-19 Pandemic?
}

\author{
Alfredo J. Ibáñez * \\ Institute for Omic Sciences and Applied Biotechnology (ICOBA PUCP), Pontificia Universidad Católica Del Perú (PUCP), Lima, \\ Peru
}

Most of us have never faced a pandemic before. The World Health Organization declared the 2019 novel coronavirus infectious disease (COVID-19), caused by the severe acute respiratory syndrome coronavirus 2 (SARS-CoV-2 virus), a pandemic by March 11th, 2020. Today, this illness has reported more than $5^{\prime} 331,019$ fatalities worldwide (December 17th, 2021). The COVID-19 pandemic has posed an unprecedented global challenge and put the academic community on "the spot." The following mini-review reports how the MS community improved the understanding of the SARS-CoV-2 virus pathophysiology while developing diagnostic procedures to complement the PCR-based approaches. For example, MS researchers identified the interaction sites between the SARS-CoV-2 virus and their hosts; this new knowledge is critical for developing antiviral drugs. MS researchers also realized that COVID-19 should be considered a systemic disease and not just a respiratory illness since its metabolic, lipidomic, and proteomic profile reflects four

OPEN ACCESS

Edited by:

Ling Lin,

Xiamen University Affiliated Cardiovascular Hospital, China

Reviewed by: Kundlik Gadhave,

Johns Hopkins University, United States

*Correspondence: Alfredo J. Ibáñez aibanez@pucp.edu.pe

Specialty section: This article was submitted to Biomedical Analysis and Diagnostics, a section of the journal Frontiers in Analytical Science

Received: 30 December 2021 Accepted: 14 February 2022

Published: 04 March 2022

Citation:

Ibáñez AJ (2022) How Is Mass Spectrometry Tackling the COVID-19

Pandemic?

Front. Anal. Sci. 2:846102. doi: 10.3389/frans.2022.846102 different clinical disorders: 1) acute inflammatory response, 2) a cardiovascular disease, 3) a prediabetic/diabetes and 4) liver dysfunction. Furthermore, MS researchers put forth the knowledge that the metabolic and lipidomic profile of several patients remained altered after being discharged, thus hinting at the scientific basis for the long COVID syndrome.

Keywords: COVID-19, SARS-CoV-2, mass spectrometry, Covid19-MSC, omics analyses

\section{INTRODUCTION}

The severe acute respiratory syndrome coronavirus 2 (SARS-CoV-2) is a member of the Coronaviridae family (Feng et al., 2020; Keni et al., 2020; Machhi et al., 2020; Tse et al., 2020; Wiersinga et al., 2020). Other coronaviruses are the severe acute respiratory syndrome coronavirus (SARS-CoV) and the middle-east respiratory syndrome-related coronavirus (MERS-CoV). Unfortunately, compared with SARS-CoV and MERS-CoV, the SARS-CoV-2 virus is highly contagious (Keni et al., 2020; Machhi et al., 2020; Tse et al., 2020; Hu et al., 2021). The SARSCoV-2 virus is the cause of the coronavirus 2019 (COVID-19) disease (Feng et al., 2020; Keni et al., 2020; Machhi et al., 2020; Tse et al., 2020; Wiersinga et al., 2020; Hu et al., 2021), which was first reported in Wuhan (Hubei Province, China) in December 2019 (Feng et al., 2020; Keni et al., 2020; Machhi et al., 2020; Tse et al., 2020; Wiersinga et al., 2020; Hu et al., 2021).

The COVID-19 human challenge study revealed that only $89 \%$ of infected participants showed symptoms (Killingley et al., 2022). Interestingly, researchers have also discussed the SARS-CoV-2 virus origin and propagation (Medema et al., 2020; Morens et al., 2020; Platto et al., 2020; Tiwari et al., 2020; La Rosa et al., 2021); some discovered that the SARS-CoV-2 virus had circulated in several countries before their first local case was reported (Medema et al., 2020; La Rosa et al., 2021). Thus, making it clear that the SARS-CoV-2 virus is difficult to contain. On March 11th, 2020, the World Health Organization (WHO) declared COVID-19 a pandemic due to its rapid spread 
worldwide (Machhi et al., 2020). According to the WHO, more than $271^{\prime} 963,258$ million cases have been reported worldwide, having thus far resulted in 5'331,019 deaths (World Health Organization, 2021).

In 2020, the mass spectrometry (MS) community formed the COVID-19 MS coalition (Covid19-MSC) (Struwe et al., 2020). MS-based technologies are especially suited for uncovering information for precision medicine, i.e., discovering biomarkers in a non-targeted and unbiased manner for disease diagnostic and prognosis. Thus, there has been a surge in the development of MS-based strategies for diagnosing COVID-19 disease from an exhaled breath, a nasopharyngeal swab, or a gargle solution (Cardozo et al., 2020; Ihling et al., 2020; Nachtigall et al., 2020; Ruszkiewicz et al., 2020; Bankar et al., 2021; Chen et al., 2021; Maus et al., 2021; Renuse et al., 2021; Tran et al., 2021). Furthermore, an exciting research line has focused on identifying biomarkers that reflect the severe COVID-19 phenotype (Chen et al., 2020a; Gordon et al., 2020; Kimhofer et al., 2020; Messner et al., 2020; Shen et al., 2020; Wu et al., 2020; Chevrier et al., 2021; Holmes et al., 2021; Lee et al., 2021; Messner et al., 2021; Wierbowski et al., 2021; Zhang et al., 2021).

Still and despite this progress, the full potential of MS applications against the COVID-19 pandemic remains to be seen. While previous MS-based reviews have zoomed in primarily on how MS approaches complement other types of diagnostics (Mahmud and Garrett, 2020; SoRelle et al., 2020; Appiasie et al., 2021; Yuan and Hu, 2021; Zhong et al., 2021; Amiri-Dashatan et al., 2022; Lima et al., 2022; Spick et al., 2022), in the following paragraphs, we will also showcase examples of MS-based strategies focused on improving our understanding of the SARS-CoV-2 virus' pathophysiology.

\section{MS for COVID-19 Detection}

Once the SARS-CoV-2 virus was sequenced and made available, real-time quantitative reverse transcription-polymerase chain reaction (RT-qPCR) and digital droplet polymerase chain reaction (dd-PCR) became the gold-standard methods of diagnosing COVID-19 (Feng et al., 2020; Walsh et al., 2020; Wiersinga et al., 2020; Hammerling et al., 2021). Unfortunately, during the beginning of the pandemic, the supply chain for these assays was inconsistent (SoRelle et al., 2020; Hammerling et al., 2021). Thus many researchers had to develop alternative strategies for SARS-CoV-2 virus detection (Cardozo et al., 2020; Grant et al., 2020; Ihling et al., 2020; Nachtigall et al., 2020; Ruszkiewicz et al., 2020; Bankar et al., 2021; Chen et al., 2021; Maus et al., 2021; Renuse et al., 2021; Tran et al., 2021; Lin et al., 2022; Mou et al., 2022).

One of Covid19-MSC's goals is to develop diagnostic procedures to complement the PCR-based approaches (Struwe et al., 2020). These MS-based strategies will possess poorer detection limits-samples must have a higher viral load $\left(10^{5}-10^{6}\right.$ genome copies per $\left.\mathrm{mL}\right)$ - than PCR-based assays (10 to $10^{2}$ genome copies per $\mathrm{mL}$ ) (SoRelle et al., 2020). The reason is that MS-based approaches lack the amplification step used in PCR-based assays (i.e., polymerase chain reaction). Nevertheless, the developed MS-based methods can still appeal to some laboratories (Cardozo et al., 2020; Ihling et al., 2020;
Nachtigall et al., 2020; Ruszkiewicz et al., 2020; Bankar et al., 2021; Chen et al., 2021; Maus et al., 2021; Renuse et al., 2021; Tran et al., 2021). Examples of such approaches are:

(a) Measurement of volatile organic compounds from breath samples (Ruszkiewicz et al., 2020; Chen et al., 2021). In this approach, exhaled breath samples are collected in tubes or bags. The samples are later injected into a gas chromatographer coupled with an ion mobility spectrometer (GC-IMS). The MS data is subsequently processed using machine-learning algorithms and other statistical tools; and

(b) Identification of a protein/peptide pattern. There are two variations to this approach:

(i) Nasal secretion samples are analyzed with a matrix-assisted laser/desorption ionization mass spectrometer (MALDI-MS) (Nachtigall et al., 2020; Tran et al., 2021). In this case, the sample is extracted using a nasopharyngeal swab. Subsequently, the swab is placed in a sterile tube with a viral transport medium. This solution is then spotted on a MALDI steel plate mixed with $a$-CHCA matrix solution and analyzed. The MS data is later processed using machinelearning algorithms; and

(ii) Nasal secretion or gargle samples are analyzed using a liquid chromatographer coupled mass spectrometer (LC-MS) (Cardozo et al., 2020; Ihling et al., 2020; Bankar et al., 2021; Maus et al., 2021; Renuse et al., 2021). The protein sample is collected using a nasopharyngeal swab or taken from a (gargle) solution. The proteins are then precipitated, digested, desalted, and measured using an LC-MS instrument, following a data-dependent acquisition (DDA) or a targeted multiple reaction monitoring (MRM) strategy.

While the metabolomics-based (i.e., GC-IMS) strategy detects the host's response to the viral infection, the proteomics-based approach can directly detect the SARS-CoV-2 viral infection in the host (i.e., viral proteins). Independently of the approach, the reported sensitivity may not be sufficient to diagnose patients at an early infection stage (SoRelle et al., 2020; Walsh et al., 2020) (Figure 1). Nevertheless, MS remains a promising tool to diagnose the COVID-19 severity by monitoring the host's proteome, metabolome, and/or lipidome after infection.

\section{MS for Understanding the COVID-19 Disease}

Successful pathogen adaptation to the host's metabolic landscape is a prerequisite for a strong viral replication (Sauer and Zamboni, 2008; Ayres, 2020; Harrison et al., 2020; Aggarwal et al., 2021; Filbin et al., 2021). Understanding the host's metabolic network changes induced by the SARS-CoV-2 viral infection is valuable for the subsequent prognosis and treatment of COVID-19 (Ayres, 2020; Tse et al., 2020; Wiersinga et al., 2020; Aggarwal et al., 2021; Filbin et al., 2021).

Our state-of-the-art knowledge about the SARS-CoV-2's disease is that the SARS-CoV-2 virus is more stable than the SARS-CoV virus (Van Doremalen et al., 2020) and has a more 
MS-based approaches for COVID-19 detection (state-of-the-art = proof-of-concept)

For diagnostic of asymptomatic patients

- MS-based diagnostics, particularly those techniques that indirectly detect the virus (by monitoring the host response), may lack the required sensitivity to identify asymptomatic patients compared to PCRbased technologies, which can directly measure lower amounts of viral copies in patients.

For diagnostic of symptomatic patients

- MS-based approaches combined with machine-learning algorithms may be used to diagnose symptomatic patients. Nevertheless, their sensitivity and selectivity need to be validated in multicentre studies and large patient cohorts before being used routinely in clinical settings.

\section{MS-based approaches for understanding the COVID-19 disease (state-of-the-art = aiming for large-scale validation)}

Based on MS-based studies, COVID-19 should be considered a systemic disease consisting of:

(i) Abnormal and unbalanced innate immune response where the signal pathways disturbed are:

- Altered proteomic/metabolomic/lipidomic profiles in plasma associated with complement activation, macrophage activation, platelet degranulation, and neutrophil degranulation.

For example, altered levels of phenylalanine and phenylalanine-related metabolites, reduced levels of glutamine, and elevated levels of glutamate in plasma. In addition to elevated levels (upregulation) of proinflammatory cytokines and chemokines, such as IL-6 and TNF.

(ii) Cardiovascular disease where the altered biological processes are:

- Altered levels of phenylalanine that correlate with markers for cardiovascular problems, such as C-reactive protein (CRP) and interleukin-6.

- Elevated mannose levels in plasma that can be associated with complement activation.

- Abnormally high levels of lipids in the blood.

(iii) Prediabetic/Diabetic phenotype based on:

- Elevated levels of sugars in the blood.

(iv) Liver dysfunction due to:

- Altered urea metabolism (i.e., elevated levels of taurine, reduced levels of citrulline, and depleted levels of carbamoyl phosphate in plasma). In addition to tryptophan-kynurenine pathway dysfunction (i.e., elevated levels of quinolinic acid, kynurenine, and 3-hydroxykynurenine in plasma).

FIGURE 1 | Summary of the MS-based approaches for COVID-19 detection and for understanding the COVID-19 disease.

flexible spike-protein that facilitates human cells infection (Turoňová et al., 2020; Ahn et al., 2021). Bioinformatic calculations (Cava et al., 2020; Wierbowski et al., 2021) and affinity purification mass spectrometry (Gordon et al., 2020; Wierbowski et al., 2021) were two key technologies that helped scientists identify SARS-CoV-2 proteins (and their active sites) that interact with their host during its life cycle and identify therapeutic targets for developing antiviral drugs to treat COVID-19 patients (Gordon et al., 2020). For example, antiviral compounds against COVID-19 target the active sites of enzymes involved in the virus's replication cycle (Mehta et al., 2020; Riva et al., 2020; Shannon et al., 2020; Shi and Puyo, 2020; Wang et al., 2020; Bakowski et al., 2021).

We also know that the clinical outcome of the SARS-CoV-2 viral infection can be highly diverse (Feng et al., 2020; Wiersinga et al., 2020; Hu et al., 2021). The result can range from the host being an asymptomatic or not-severe patient (i.e., concludes with a fast and full recovery) to a severe patient (i.e., suffers from various complications) (Docherty et al., 2020; Guan et al., 2020; Munayco et al., 2020). These complications can lead to organ dysfunction and death due to an abnormal and unbalanced immune response, known as sepsis. Thus, an additional goal of Covid19-MSC was to complement the PCR-based diagnostics, which cannot predict the severity of the strains, by defining clinical phenotypes of interest and monitoring patient treatment/recovery (Struwe et al., 2020).
Before reviewing the excellent work done by researchers to understand the COVID-19 pathogenesis, it is crucial to mention common limitations that all these scientists expressed in their publication:

1) One limitation was the size of the patient cohorts in some studies (i.e., less than 100 patients). Hence, the authors validated their hypothesis with available published studies by other research groups.

2) Another challenge was correlating a particular MS signal profile with a specific clinical phenotype, such as COVID19 severity. Especially when circulating proteins, metabolites, and lipids from blood or plasma samples may have multiple sources (e.g., comorbidities). Thus, authors use alternative methods to validate their results (i.e., multi-omics data analysis).

3) When trying to find markers for COVID-19 disease severity, the authors considered that severe COVID-19 patients were usually older or had additional clinical risk factors than mild COVID-19 patients. Furthermore, they also thought of the skewing of the data in favor of sicker patients at later timepoints since mild COVID-19 patients are less likely to stay hospitalized for several days than severe COVID-19 patients.

Messner et al. identified a plasma proteome signature (24 proteins) differently expressed depending on COVID-19 severity 
in two independent studies with different population sizes using an ultra-high-performance liquid chromatography/tandem mass spectrometry (UHPLC-MS/MS) (Messner et al., 2020; Messner et al., 2021). These proteins were associated with the complement system and the inflammatory response (i.e., several inflammation modulators). The protein signature allowed them to reclassify a suspected COVID-19 patient suffering from an influenza type B infection, showing the potential of the UHPLC-MS/MS method to support clinical decision-making (Messner et al., 2020).

Using a stable isotope-labeled nano-liquid chromatography coupled to mass spectrometry (nLC-MS) proteomic strategy, Shen et al. identified 93 blood sera proteins correlated with severe COVID-19 patients (Shen et al., 2020). From these 93 proteins, 50 proteins belong to three major pathways: 1) complement system, 2) macrophage activation, and 3) platelet degranulation. They verified their results in an additional cohort of patients and performed a non-targeted metabolomic study. The metabolomic study showed 80 metabolites that significantly changed with COVID-19 severity and were involved in the three biological processes revealed in the proteomic analysis. Thus, the authors proposed a classifier for COVID-19 severity based on monitoring 22 serum proteins and 7 metabolites in patient serum. Although the overall classifier achieved an accuracy of $93.5 \%$ in the training set, it misclassified a few patients, reflecting the complexity of the clinical cohort. Nevertheless, it was able to classify five severe patients 1-4 days before they were clinically diagnosed as severe patients.

Interestingly, the correlation between COVID-19 severity and the macrophage activation and complement activation was confirmed by a single-cell mass cytometry clinical study and MRM-based assay. Chevrier et al. (Chevrier et al., 2021) showed using single-cell mass cytometry that mild and severe disease patients showed a similar composition of myeloid cells during the early symptom stage. Nevertheless, a stronger inflammatory phenotype is observed in patients experiencing severe symptoms during the later stages of the disease, i.e., CD169 monocytes and higher pro-inflammatory cytokines. The work of Bankar et al. showed using an MRM strategy an increase in peripheral neutrophil degranulation and the increase of proinflammatory cytokines (Bankar et al., 2021). Neutrophil degranulation may induce complement activation (Camous et al., 2011; Bankar et al., 2021) to eliminate the SARS-CoV-2 virus. Nevertheless, an unbalanced release of granule-derived mediators may lead to septic shock (Lacy, 2006). Thus, Bankar et al. pointed out that it is unclear whether SARS-CoV-2 directly targets the neutrophil degranulation pathway or is just a consequence of the SARS-CoV-2 complications (Bankar et al., 2021).

The increment of pro-inflammatory cytokines can dysregulate lipid metabolism and vascular permeability (Calder, 2002; Aslani et al., 2021). Zhang et al. explored this concept by monitoring the levels of serum proteins during the progression of the COVID-19 disease using a SWATH-MS (i.e., UHPLC-MS/MS) workflow combined with machine learning (Zhang et al., 2021). Their study found that low-density lipoproteins (LDLs) and other apolipoproteins significantly decrease in COVID-19 patients, possibly due to pro-inflammatory cytokines. Hence, they propose that serum protein levels of proteins involved in lipid metabolism can be used as a potential predictor of the prognosis in COVID-19 patients.

Additionally to the proteome, the metabolome and lipidome in COVID-19 patients vary with infection and could be correlated to the severity of the SARS-CoV-2 viral infection. Wu et al. observed altered metabolic and lipidomic profiles using an LCMS system (Wu et al., 2020). These profiles proved that SARSCoV-2 hijacks the host cell's nucleic acids biosynthetic metabolic pathways (i.e., biosynthesis of purine and pyrimidine nucleotides) and its ability to balance its energy metabolism (i.e., TCA cycle) (Wu et al., 2020).

$\mathrm{Wu}$ et al. also observed that guanosine monophosphate (GMP) and carbamoyl phosphate were depleted in COVID-19 positive patients. Since GMP production depends on enzymes that have a role in the immune system (Wu et al., 2020), and carbamoyl phosphate is synthesized by enzymes in the urea metabolism (Strick-Marchand et al., 2004; Wu et al., 2020), the authors proposed that COVID-19 patients might suffer from immune and liver dysfunction (Wu et al., 2020), respectively; in addition to the possibility of cardiovascular complications due to the abnormally high levels of lipids in their blood (Kris-Etherton, 1999; Wu et al., 2020).

Lee et al. used a combination of gas chromatography-mass spectrometry (GC-MS) and UHPLC-MS/MS to analyze plasma samples (Lee et al., 2021). They also used a cell sorter for better classifying disease severity and predicting clinical outcomes by performing their metabolomic analysis on a homogenous cellular population. The authors observed two independent modes of metabolic reprogramming due to the SARS-CoV-2 viral infection. The first corresponds to changes in the quantity of the metabolically active immune cell subpopulations, while the second involves shifts in the metabolism within individual cells within a subpopulation. By doing so, the authors observed that metabolites (e.g., phenylalanine) that are correlated with proinflammatory cytokines are also positively correlated with COVID-19 severity. In contrast, other metabolites and lipids (particularly those associated with cytokine synthesis) were negatively correlated with the disease severity (Lee et al., 2021). The observed profiles by Lee et al. are similar to those identified by Meoni et al. (Meoni et al., 2021) using an NMRbased approach. Lee et al. (Lee et al., 2021) and Meoni et al. (Meoni et al., 2021) correlate these changes to an inflammation and immune activation response against COVID-19.

Lee et al. also detected high plasma levels of mannose and glucose that correlated with the severity of the COVID-19 disease. They suggested two hypotheses 1) that mannose levels in plasma can be derived from residues of SARS-CoV-2 spike protein, potentially reflecting high viral loads; and 2) that the high mannose levels in plasma may indicate the complement pathway activation. Although the latter explanation has been proposed by other authors (Camous et al., 2011; Zhang et al., 2021), Lee et al. expressed that this profile is also consistent with patients suffering from coronary heart disease (Jones et al., 1999; Murr et al., 2014; Chen et al., 2020b; Lee et al., 2021).

Chen et al. demonstrated that significant changes in the levels of lipoprotein subclasses and their compositional components are 
correlated with COVID-19 severity using a combination of nLCMS and nuclear magnetic resonance (NMR) techniques (Chen et al., 2020a). For example, levels of triglycerides (TG) in lowdensity lipoprotein subclass 1 (LDL 1) and free cholesterol (FC) in all very-low-density lipoprotein subclass 5 were significantly elevated in both mild and severe patients when compared with healthy controls. Moreover, key proteins involved in lipoprotein and related metabolic pathways were elevated considerably or reduced beyond typical healthy values (as shown by Zhang et al. (Zhang et al., 2021) and Wei et al. (Wei et al., 2020)). Fortunately, most enzymes and lipoprotein levels recovered when the patients were discharged, thus showing a transient behavior. Therefore, Chen et al. propose that during SARS-CoV-2 infection, there is a significant dysregulation in lipoprotein metabolism (e.g., hypolipidemia), glycolysis, and TCA cycle (Chen et al., 2020a).

Kimhofer et al. also performed a deep UHPLC-MS/MS and NMR-based metabolomic and lipidomic study on plasma samples (Kimhofer et al., 2020). They observed metabolomic and lipidomic profiles that other authors correlated with four different clinical disorders: 1) acute inflammatory response (Kimhofer et al., 2020; Meoni et al., 2021), 2) a cardiovascular risk signature (Kris-Etherton, 1999; Kimhofer et al., 2020), 3) a prediabetic/diabetes-like signature (Krauss, 2004; Kimhofer et al., 2020), and 4) liver dysfunction (Kopple, 2007; Kimhofer et al., 2020). The authors described that these metabolic disturbances appeared independently of the severity of the respiratory symptoms or the exact sampling time-point with respect to the onset of the COVID-19 symptoms (Kimhofer et al., 2020).

Interestingly, Kimhofer et al. (Kimhofer et al., 2020) reminded us that patients who had recovered from SARS-CoV-1 infection had further complications such as hyperlipidemia, cardiovascular abnormalities, and glucose metabolism disorders. Concerning this point, $\mathrm{Wu}$ et al. (Wu et al., 2020) and Kimhofer et al. (Kimhofer et al., 2020) argue that COVID-19 should be considered a systemic disease and not just a respiratory illness. This sentiment is echoed by other COVID-19 independent studies (Gupta et al., 2020; Nalbandian et al., 2020; Wang et al., 2021a; Duan et al., 2021; Frontera et al., 2021; LopezLeon et al., 2021; Sanchez-Vazquez et al., 2021). Furthermore, many authors (Kimhofer et al., 2020; Zhu et al., 2020; Logette et al., 2021) make a case that comorbidities will complicate the patients' treatment and should be addressed and managed as early as possible to avoid long-term complications that have recently been described as "long COVID syndrome." Therefore, MS techniques may be required to monitor postcovid patients, since although mild and severe patients diagnosed with COVID-19 had met the official hospital discharge criteria (i.e., COVID-19 nucleic acid tests were negative, and many clinical signs had disappeared), many levels of proteins, metabolites, and lipids had not returned to normal by the time they were discharged (Balachandar et al., 2020; Wu et al., 2020; Holmes et al., 2021).

Holmes et al. used an NMR and UHPLC-MS/MS-based approach to monitor the blood plasma samples to understand the long COVID syndrome (Holmes et al., 2021). For this study, the authors defined three different cohorts: 1) a healthy control group, 2) a hospitalized patient group sampled during the acute infection phase), and 3) a recovery cohort consisting of a nonhospitalized group. The latter group was sampled 3 months post the acute phase (hospitalization stage) and 6 months post their tentative date of COVID-19 infection. Thus, the authors assessed the phenoconversion, i.e., the change from a standard (i.e., healthy) phenotype to an altered (i.e., sick) phenotype. Furthermore, they were able to identify the metabolic profiles of patients suffering from long COVID-19, i.e., with incomplete functional recovery. Interestingly, $57 \%$ of the participants recorded one or more persistent symptoms within the recovery cohort. The majority had more than one symptom not associated with the respiratory system.

As in the works of Wu et al. (Wu et al., 2020) and Kimhofer et al. (Kimhofer et al., 2020), Holmes et al. identified that while metabolic and lipoprotein parameters which were altered during SARS-CoV-2 infection returned to a healthy range, other parameters such as the glutamine/glutamate ratio, which is essential for immune cell homeostasis, remained altered (Holmes et al., 2021). Unfortunately, they could not provide a mechanistic significance to the glutamine/glutamate ratio during the acute and post-acute infection stages with SARS-CoV-2. Nevertheless, they propose that this low glutamine/glutamate ratio implies a continuing post-COVID immune dysregulation.

Other altered metabolic patterns observed by the authors were: 1) elevated taurine and low citrulline (associated with liver dysfunction) (Yu et al., 2017; Holmes et al., 2021), 2) high quinolinic acid, kynurenine, 3-hydroxykynurenine (associated with inflammation and liver dysfunction) (Heyes et al., 1997; Holmes et al., 2021), and 3) increased levels of 3-indole-acetic acid, which may imply a microbiome functionality shift in recovered COVID-19 patients (Blasco et al., 2020; Holmes et al., 2021). Nevertheless, due to the high degree of interindividual variability (age and comorbidities) in the follow-up patients, the authors expressed that the long-term clinical significance of these observations will require further investigation (Holmes et al., 2021).

Although the exact patterns of altered biomarkers (proteins, lipids and metabolites) were not identical in all reviewed publications, the data shows that COVID-19 disease is a mixture of four different clinical disorders (Figure 1): 1) inflammation and cell death triggered by the innate immune response (Chen et al., 2020a; Kimhofer et al., 2020; Messner et al., 2020; Shen et al., 2020; Wu et al., 2020; Bankar et al., 2021; Chevrier et al., 2021; Holmes et al., 2021; Lee et al., 2021; Messner et al., 2021; Zhang et al., 2021); 2) a cardiovascular disease (Kimhofer et al., 2020; Wu et al., 2020; Lee et al., 2021), 3) a prediabetic/diabetes-like disease (Kimhofer et al., 2020; Wu et al., 2020), and 4) liver dysfunction (Kimhofer et al., 2020; Wu et al., 2020; Holmes et al., 2021; Lee et al., 2021). Furthermore, severe COVID-19 cases show a temporally delayed activation of monocyte pathways and an increased expression over time of pro-inflammatory cytokines, which may lead to a septic shock (Bankar et al., 2021; Chevrier et al., 2021). These profiles can be the basis for developing clinical testing for COVID-19 severity prognosis, which relies on targeted strategies using reliable and low-cost effective (accessible) instrumentation. Examples of such diagnostic methods that can be used to determine the severity of 
COVID-19 patients are image-based diagnostics looking for lung inflammation (Docherty et al., 2020; Guan et al., 2020; Liu et al., 2020; Okolo et al., 2021) and blood/urine-based approaches looking for inflammation, cardiovascular, diabetes-type, and liver dysfunction biomarkers (Gross et al., 2020; Gross et al., 2021; Siemens Healthineers. Lev, 2021).

The reviewed data also shows that discharged patients still present an incomplete functional recovery, i.e., long COVID (Kimhofer et al., 2020; Liu et al., 2020; Nalbandian et al., 2020; Wu et al., 2020; Holmes et al., 2021; Lopez-Leon et al., 2021; Taquet et al., 2021; Xie et al., 2022). Mobile apps could help monitor better long COVID symptoms (Menni et al., 2020; Wise, 2020; Chang et al., 2021; Louca et al., 2021). For example, a Peruvian mobile app (ARIM) is used by medical personnel to register clinical data from patients (Characterizing COVID-19, 2020; ARIM 2.0, 2021). If used uniformly at a regional/national level, ARIM and similar apps can provide anonymized data for early warnings of an epidemic infection outbreak (wave) and improve our understanding of the long COVID disease symptomology (SoRelle et al., 2020).

\section{CONCLUSION}

It is uncertain how the COVID-19 pandemic will develop (Clark et al., 2020; Gandhi et al., 2020; Korber et al., 2020; Long et al., 2020; Almufarrij and Munro, 2021; Wang et al., 2021b; Gao et al., 2021; Hodcroft et al., 2021; Karim and Karim, 2021; McCallum et al., 2021; Peacock et al., 2021; Subramanya et al., 2021; Thomson et al., 2021;

\section{REFERENCES}

Aggarwal, S., Acharjee, A., Mukherjee, A., Baker, M. S., and Srivastava, S. Role of Multiomics Data to Understand Host-Pathogen Interactions in COVID-19 Pathogenesis. J. Proteome Res. (2021) 20(2):1107-1132. doi:10.1021/acs. jproteome.0c00771

Ahn, J. H., Kim, J., Hong, S. P., Choi, S. Y., Yang, M. J., Ju, Y. S., et al. Nasal Ciliated Cells Are Primary Targets for SARS-CoV-2 Replication in the Early Stage of COVID-19. J. Clin. Invest. (2021) 131(13):e148517. doi:10. $1172 /$ JCI1 48517

Almufarrij, I., and Munro, K. J. One Year on: an Updated Systematic Review of SARS-CoV-2, COVID-19 and Audio-Vestibular Symptoms. Int. J. Audiol. (2021) 60(12):935-945. doi:10.1080/14992027.2021.1896793

Amiri-Dashatan, N., Koushki, M., and Rezaei-Tavirani, M. Mass SpectrometryBased Proteomics Research to Fight COVID-19: An Expert Review on Hopes and Challenges. OMICS: A J. Integr. Biol. (2022) 26(1):19-34. doi:10.1089/omi. 2021.0182

Appiasie, D., Guerra, D. J., Tanguay, K., Jelinek, S., Guerra, D. D., and Sen, R. "Multiomics" Approaches to Understand and Treat COVID-19: Mass Spectrometry and Next-Generation Sequencing. BioChem. (2021) 1(3): 210-237. doi:10.3390/biochem1030016

ARIM 2.0. Application for the Registration of Medical Information to Support the Health Sector during the COVID-19 Pandemic in Peru [No 025-2021FONDECYT] Was Funded by CONCYTEC-FONDECYT within the Framework of the Special Projects: Modality - Scaling of Diagnostic Kits, Devices and Medical Technologies for COVID-19 (2021).

Aslani, M., Mortazavi-Jahromi, S. S., and Mirshafiey, A. Cytokine Storm in the Pathophysiology of COVID-19: Possible Functional Disturbances of miRNAs. Int. Immunopharmacology (2021) 101(Pt A):108172. doi:10.1016/j.intimp. 2021.108172
Ward et al., 2021; Garcia-Beltran et al., 2022; Katzourakis, 2022; Konrath et al., 2022). In retrospect, the scientific community managed to rise to the challenge (Rijs and Fenter, 2020). Compared to PCR-based COVID-19, MS-based strategies are less sensitive. Nevertheless, mass spectrometry can identify the metabolomic, lipidomic, and proteomic profiles associated with COVID-19 disease severity. These profiles provide valuable information on the underlying biological processes responsible for the severe disease phenotype and can be the basis for designing costeffective diagnostics of COVID-19 severity. Interestingly, imagebased and blood/urine-based approaches have already been validated in multicenter studies. We hope that the Covid19-MSC initiative will catalyze in the near future multicenter initiatives to validate targeted MS-based quantification of biomarkers to determine COVID-19 disease severity.

\section{AUTHOR CONTRIBUTIONS}

Author Contributions Conceptualization: AI Data curation: AI Writing - original draft, review and editing: AI.

\section{FUNDING}

The research of AI is supported by "The Max Planck Partner Group," and CONCYTEC: “ARIM 2.0: Aplicación para el registro de información médica para apoyar el sector salud durante la pandemia COVID-19 en Perú" (025-2021)."

Ayres, J. S. A Metabolic Handbook for the COVID-19 Pandemic. Nat. Metab. (2020) 2(7):572-585. doi:10.1038/s42255-020-0237-2

Bakowski, M. A., Beutler, N., Wolff, K. C., Kirkpatrick, M. G., Chen, E., Nguyen, T. T., et al. Drug Repurposing Screens Identify Chemical Entities for the Development of COVID-19 Interventions. Nat. Commun. (2021) 12(1): 3309. doi:10.1038/s41467-021-23328-0

Balachandar, V., Mahalaxmi, I., Subramaniam, M., Kaavya, J., Senthil Kumar, N., Laldinmawii, G., et al. Follow-up Studies in COVID-19 Recovered Patients - Is it Mandatory? Sci. Total Environ. (2020) 729:139021. doi:10.1016/j.scitotenv. 2020.139021

Bankar, R., Suvarna, K., Ghantasala, S., Banerjee, A., Biswas, D., Choudhury, M., et al. Proteomic Investigation Reveals Dominant Alterations of Neutrophil Degranulation and mRNA Translation Pathways in Patients with COVID-19. iScience (2021) 24(3):102135. 102135. doi:10.1016/j.isci.2021.102135

Blasco, H., Bessy, C., Plantier, L., Lefevre, A., Piver, E., Bernard, L., et al. The Specific Metabolome Profiling of Patients Infected by SARS-COV-2 Supports the Key Role of Tryptophan-Nicotinamide Pathway and Cytosine Metabolism. Sci. Rep. (2020) 10(1):16824. 16824. doi:10.1038/s41598-020-73966-5

Calder, P. C. Dietary Modification of Inflammation with Lipids. Proc. Nutr. Soc. (2002) 61(3):345-358. doi:10.1079/pns2002166

Camous, L., Roumenina, L., Bigot, S., Brachemi, S., Frémeaux-Bacchi, V., Lesavre, P., et al. Complement Alternative Pathway Acts as a Positive Feedback Amplification of Neutrophil Activation. Blood (2011) 117(4):1340-1349. doi:10.1182/blood-2010-05-283564

Cardozo, K. H. M., Lebkuchen, A., Okai, G. G., Schuch, R. A., Viana, L. G., Olive, A. N., et al. Establishing a Mass Spectrometry-Based System for Rapid Detection of SARS-CoV-2 in Large Clinical Sample Cohorts. Nat. Commun. (2020) 11(1): 6201. doi:10.1038/s41467-020-19925-0

Cava, C., Bertoli, G., and Castiglioni, I. A Protein Interaction Map Identifies Existing Drugs Targeting SARS-CoV-2. BMC Pharmacol. Toxicol. (2020) 21(1): 65. 65. doi:10.1186/s40360-020-00444-Z 
Chang, S., Pierson, E., Koh, P. W., Gerardin, J., Redbird, B., Grusky, D., et al. Mobility Network Models of COVID-19 Explain Inequities and Inform Reopening. Nature (2021) 589(7840):82-87. doi:10.1038/s41586-020-2923-3

Characterizing COVID-19. COVID-19 Patient Data Analysis Tool [035-2020FONDECYT] Was Funded by CONCYTEC-FONDECYT within the Framework of the Special Projects: Response to the COVID-19 (2020). call 01.

Chen, H., Qi, X., Zhang, L., Li, X., Ma, J., Zhang, C., et al. COVID-19 Screening Using Breath-Borne Volatile Organic Compounds. J. Breath Res. (2021) 15(4). doi:10.1088/1752-7163/ac2e57

Chen, W. S., Wang, C. H., Cheng, C. W., Liu, M. H., Chu, C. M., Wu, H. P., et al. Elevated Plasma Phenylalanine Predicts Mortality in Critical Patients with Heart Failure. ESC Heart Fail. (2020) 7(5):2884-2893. doi:10.1002/ ehf2.12896

Chen, Y. M., Zheng, Y., Yu, Y., Wang, Y., Huang, Q., Qian, F., et al. Blood Molecular Markers Associated with COVID-19 Immunopathology and MultiOrgan Damage. EMBO J. (2020) 39(24):e105896. e105896. doi:10.15252/embj. 2020105896

Chevrier, S., Zurbuchen, Y., Cervia, C., Adamo, S., Raeber, M. E., de Souza, N., et al. A Distinct Innate Immune Signature marks Progression from Mild to Severe COVID-19. Cell Rep Med (2021) 2(1):100166. 100166. doi:10.1016/j.xcrm.2020. 100166

Clark, A., Jit, M., Warren-Gash, C., Guthrie, B., Wang, H. H. X., Mercer, S. W., et al. Global, Regional, and National Estimates of the Population at Increased Risk of Severe COVID-19 Due to Underlying Health Conditions in 2020: a Modelling Study. Lancet Glob. Health (2020) 8(8):e1003-e1017. doi:10.1016/S2214109X(20)30264-3

Docherty, A., Harrison, E., Green, C., Hardwick, H., Pius, R., Norman, L., et al. Features of 16,749 Hospitalized UK Patients with COVID-19 Using the ISARIC WHO Clinical Characterization Protocol. BMJ (2020) 369. doi:10.1136/bmj. m1985

Duan, K., Premi, E., Pilotto, A., Cristillo, V., Benussi, A., Libri, I., et al. Alterations of Frontal-Temporal gray Matter Volume Associate with Clinical Measures of Older Adults with COVID-19. Neurobiol. Stress (2021) 14:100326. doi:10.1016/ j.ynstr.2021.100326

Feng, W., Newbigging, A. M., Le, C., Pang, B., Peng, H., Cao, Y., et al. Molecular Diagnosis of COVID-19: Challenges and Research Needs. Anal. Chem. (2020) 92:10196-10209. doi:10.1021/acs.analchem.0c02060

Filbin, M. R., Mehta, A., Schneider, A. M., Kays, K. R., Guess, J. R., Gentili, M., et al. Longitudinal Proteomic Analysis of Severe COVID-19 Reveals SurvivalAssociated Signatures, Tissue-specific Cell Death, and Cell-Cell Interactions. Cel Rep Med (2021) 2(5):100287. 100287. doi:10.1016/j.xcrm.2021.100287

Frontera, J. A., Sabadia, S., Lalchan, R., Fang, T., Flusty, B., Millar-Vernetti, P., et al. A Prospective Study of Neurologic Disorders in Hospitalized Patients with COVID-19 in New York City. Neurology (2021) 96(4):e575-e586. doi:10.1212/ WNL.0000000000010979

Gandhi, R. T., Lynch, J. B., and Del Rio, C. Mild or Moderate Covid-19. N. Engl. J. Med. (2020) 383(18):1757-1766. doi:10.1056/NEJMcp2009249

Gao, Z., Xu, Y., Sun, C., Wang, X., Guo, Y., Qiu, S., et al. A Systematic Review of Asymptomatic Infections with COVID-19. J. Microbiol. Immunol. Infect. (2021) 54(1):12-16. doi:10.1016/j.jmii.2020.05.001

Garcia-Beltran, W. F., St. Denis, K. J., Hoelzemer, A., Lam, E. C., Nitido, A. D., Sheehan, M. L., et al. mRNA-based COVID-19 Vaccine Boosters Induce Neutralizing Immunity against SARS-CoV-2 Omicron Variant. Cell. (2022) 185(3):457-466. doi:10.1016/j.cell.2021.12.033

Gordon, D. E., Jang, G. M., Bouhaddou, M., Xu, J., Obernier, K., White, K. M., et al. A SARS-CoV-2 Protein Interaction Map Reveals Targets for Drug Repurposing. Nature (2020) 583(7816):459-468. doi:10.1038/s41586-0202286-9

Grant, B. D., Anderson, C. E., Williford, J. R., Alonzo, L. F., Glukhova, V. A., Boyle, D. S., et al. SARS-CoV-2 Coronavirus Nucleocapsid Antigen-Detecting HalfStrip Lateral Flow Assay toward the Development of point of Care Tests Using Commercially Available Reagents. Anal. Chem. (2020) 92(16):11305-11309. Aug 18. doi:10.1021/acs.analchem.0c01975

Gross, O., Moerer, O., Rauen, T., Böckhaus, J., Hoxha, E., Jörres, A., et al. Validation of a Prospective Urinalysis-Based Prediction Model for ICU Resources and Outcome of COVID-19 Disease: A Multicenter Cohort Study. Jcm (2021) 10(14):3049. Jul 9. doi:10.3390/jcm10143049
Gross, O., Moerer, O., Weber, M., Huber, T. B., and Scheithauer, S. COVID-19associated Nephritis: Early Warning for Disease Severity and Complications? The Lancet (2020) 395(10236):e87-e88. doi:10.1016/S0140-6736(20)31041-2

Guan, W.-j., Ni, Z.-y., Hu, Y., Liang, W.-h., Ou, C.-q., He, J.-x., et al. Clinical Characteristics of Coronavirus Disease 2019 in China. N. Engl. J. Med. (2020) 382:1708-1720. doi:10.1056/NEJMoa2002032

Gupta, A., Madhavan, M. V., Sehgal, K., Nair, N., Mahajan, S., Sehrawat, T. S., et al. Extrapulmonary Manifestations of COVID-19. Nat. Med. (2020) 26(7): 1017-1032. doi:10.1038/s41591-020-0968-3

Hammerling, M. J., Warfel, K. F., and Jewett, M. C. Lyophilization of Premixed COVID-19 Diagnostic RT-qPCR Reactions Enables Stable Long-Term Storage at Elevated Temperature. Biotechnol. J. (2021) 16(7):e2000572. doi:10.1002/ biot. 202000572

Harrison, A. G., Lin, T., and Wang, P. Mechanisms of SARS-CoV-2 Transmission and Pathogenesis. Trends Immunol. (2020) 41(12):1100-1115. doi:10.1016/j.it. 2020.10.004

Heyes, M. P., Chen, C. Y., Major, E. O., and Saito, K. Different Kynurenine Pathway Enzymes Limit Quinolinic Acid Formation by Various Human Cell Types. Biochem. J. (1997) 326:351-356. doi:10.1042/bj3260351

Hodcroft, E. B., Zuber, M., Nadeau, S., Vaughan, T. G., Crawford, K. H. D., Althaus, C. L., et al. Spread of a SARS-CoV-2 Variant through Europe in the Summer of 2020. Nature (2021) 595(7869):707-712. doi:10.1038/s41586-021-03677-y

Holmes, E., Wist, J., Masuda, R., Lodge, S., Nitschke, P., Kimhofer, T., et al. Incomplete Systemic Recovery and Metabolic Phenoreversion in Post-AcutePhase Nonhospitalized COVID-19 Patients: Implications for Assessment of Post-Acute COVID-19 Syndrome. J. Proteome Res. (2021) 20(6):3315-3329. doi:10.1021/acs.jproteome.1c00224

Hu, B., Guo, H., Zhou, P., and Shi, Z.-L. Characteristics of SARS-CoV-2 and COVID-19. Nat. Rev. Microbiol. (2021) 19(3):141-154. doi:10.1038/s41579020-00459-7

Ihling, C., Tänzler, D., Hagemann, S., Kehlen, A., Hüttelmaier, S., Arlt, C., et al. Mass Spectrometric Identification of SARS-CoV-2 Proteins from Gargle Solution Samples of COVID-19 Patients. J. Proteome Res. (2020) 19(11): 4389-4392. doi:10.1021/acs.jproteome.0c00280

Jones, S. A., Novick, D., Horiuchi, S., Yamamoto, N., Szalai, A. J., and Fuller, G. M. C-reactive Protein: a Physiological Activator of Interleukin 6 Receptor Shedding. J. Exp. Med. (1999) 189(3):599-604. doi:10.1084/jem.189.3.599

Karim, S. S. A., and Karim, Q. A. Omicron SARS-CoV-2 Variant: a New Chapter in the COVID-19 Pandemic. The Lancet (2021) 398(10317):2126-2128. doi:10. 1016/S0140-6736(21)02758-6

Katzourakis, A. COVID-19: Endemic Doesn't Mean Harmless. Nature (2022 Jan) 601(7894):485. doi:10.1038/d41586-022-00155-X

Keni, R., Alexander, A., Nayak, P. G., Mudgal, J., and Nandakumar, K. COVID-19: Emergence, Spread, Possible Treatments, and Global burden. Front. Public Health (2020) 8:216. doi:10.3389/fpubh.2020.00216

Killingley, B., Mann, A., Kalinova, M., Boyers, A., Goonawardane, N., Zhou, J., et al. Safety, Tolerability and Viral Kinetics during SARS-CoV-2 Human challenge. Res. Square (2022). doi:10.21203/rs.3.rs-1121993/v1

Kimhofer, T., Lodge, S., Whiley, L., Gray, N., Loo, R. L., Lawler, N. G., et al. Integrative Modeling of Quantitative Plasma Lipoprotein, Metabolic, and Amino Acid Data Reveals a Multiorgan Pathological Signature of SARSCoV-2 Infection. J. Proteome Res. (2020) 19(11):4442-4454. doi:10.1021/acs. jproteome.0c00519

Konrath, K. M., Liaw, K., Wu, Y., Zhu, X., Walker, S. N., Xu, Z., et al. Nucleic Acid Delivery of Immune-Focused SARS-CoV-2 Nanoparticles Drives Rapid and Potent Immunogenicity Capable of Single-Dose protection. Cel Rep. (2022) 38(5):110318. doi:10.1016/j.celrep.2022.110318

Kopple, J. D. Phenylalanine and Tyrosine Metabolism in Chronic Kidney Failure. J. Nutr. (2007) 137:1586S-1590S. doi:10.1093/jn/137.6.1586S

Korber, B., Fischer, W. M., Gnanakaran, S., Yoon, H., Theiler, J., Abfalterer, W., et al. Tracking Changes in SARS-CoV-2 Spike: Evidence that D614G Increases Infectivity of the COVID-19 Virus. Cell. (2020) 182(4):812-827. doi:10.1016/j. cell.2020.06.043

Krauss, R. M. Lipids and Lipoproteins in Patients with Type 2 Diabetes. Diabetes Care (2004) 27(6):1496-1504. doi:10.2337/diacare.27.6.1496

Kris-Etherton, P. M. Monounsaturated Fatty Acids and Risk of Cardiovascular Disease. Circulation (1999) 100(11):1253-1258. doi:10.1161/01.cir.100.11.1253 
La Rosa, G., Mancini, P., Bonanno Ferraro, G., Veneri, C., Iaconelli, M., Bonadonna, L., et al. SARS-CoV-2 Has Been Circulating in Northern Italy since December 2019: Evidence from Environmental monitoringEvidence from Environmental Monitoring. Sci. Total Environ. (2021) 750(750):141711. December. doi:10.1016/j.scitotenv.2020.141711

Lacy, P. Mechanisms of Degranulation in Neutrophils. All Asth Clin. Immun. (2006) 2(3):98-108. doi:10.1186/1710-1492-2-3-98

Lee, J. W., Su, Y., Baloni, P., Chen, D., Pavlovitch-Bedzyk, A. J., Yuan, D., et al. Integrated Analysis of Plasma and Single Immune Cells Uncovers Metabolic Changes in Individuals with COVID-19. Nat. Biotechnol. (2021). doi:10.1038/ s41587-021-01020-4

Lima, N. M., Fernandes, B. L. M., Alves, G. F., de Souza, J. C. Q., Siqueira, M. M., Patrícia do Nascimento, M., et al. Mass Spectrometry Applied to Diagnosis, Prognosis, and Therapeutic Targets Identification for the Novel Coronavirus SARS-CoV-2: A Review. Analytica Chim. Acta (2022) 1195:339385. doi:10. 1016/j.aca.2021.339385

Lin, E. E., Razzaque, U. A., Burrows, S. A., and Smoukov, S. K. End-to-end System for Rapid and Sensitive Early-Detection of SARS-CoV-2 for Resource-Poor and Field-Test Environments Using a \$51 Lab-In-A-Backpack. PLoS One (2022) 17(1):e0259886. Jan 26e0259886. doi:10.1371/journal.pone.0259886

Liu, C., Ye, L., Xia, R., Zheng, X., Yuan, C., Wang, Z., et al. Chest Computed Tomography and Clinical Follow-Up of Discharged Patients with COVID-19 in Wenzhou City, Zhejiang, China. Ann. ATS (2020) 17(10):1231-1237. doi:10. 1513/AnnalsATS.202004-324OC10.1513/annalsats.202004-324oc

Logette, E., Lorin, C., Favreau, C., Oshurko, E., Coggan, J. S., Casalegno, F., et al. A Machine-Generated View of the Role of Blood Glucose Levels in the Severity of COVID-19. Front. Public Health (2021) 9:695139. doi:10.3389/fpubh.2021. 695139

Long, S. W., Olsen, R. J., Christensen, P. A., Bernard, D. W., Davis, J. J., Shukla, M., et al. Molecular Architecture of Early Dissemination and Massive Second Wave of the SARS-CoV-2 Virus in a Major Metropolitan Area. medRxiv (2020) 11(6): e02707-02720. doi:10.1128/mBio.02707-2010.1101/2020.09.22.20199125

Lopez-Leon, S., Wegman-Ostrosky, T., Perelman, C., Sepulveda, R., Rebolledo, P. A., Cuapio, A., et al. More Than 50 Long-Term Effects of COVID-19: a Systematic Review and Meta-Analysis. Sci. Rep. (2021) 11(1):16144. 16144. doi:10.1038/s41598-021-95565-8

Louca, P., Murray, B., Klaser, K., Graham, M. S., Mazidi, M., Leeming, E. R., et al. Modest Effects of Dietary Supplements during the COVID-19 Pandemic: Insights from 445850 Users of the COVID-19 Symptom Study App. Bmjnph (2021) 4(1):149-157. doi:10.1136/bmjnph-2021-000250

Machhi, J., Herskovitz, J., Senan, A. M., Dutta, D., Nath, B., Oleynikov, M. D., et al. The Natural History, Pathobiology, and Clinical Manifestations of SARS-CoV2 Infections. J. Neuroimmune Pharmacol. (2020) 15(3):359-386. doi:10.1007/ s11481-020-09944-5

Mahmud, I., and Garrett, T. J. Mass Spectrometry Techniques in Emerging Pathogens Studies: COVID-19 Perspectives. J. Am. Soc. Mass. Spectrom. (2020) 31(10):2013-2024. 2013-2024. doi:10.1021/jasms.0c00238

Maus, A., Renuse, S., Kemp, J., Madugundu, A. K., Vanderboom, P. M., Blommel, J., et al. Targeted Detection of SARS-CoV-2 Nucleocapsid Sequence Variants by Mass Spectrometric Analysis of Tryptic Peptides. J. Proteome Res. (2021) 21: 142-150. acs.jproteome.1c00613. doi:10.1021/acs.jproteome.1c00613

McCallum, M., Bassi, J., De Marco, A., Chen, A., Walls, A. C., Di Iulio, J., et al. SARS-CoV-2 Immune Evasion by the B.1.427/B.1.429 Variant of Concern. Science (2021) 373(6555):648-654. doi:10.1126/science.abi7994

Medema, G., Heijnen, L., Elsinga, G., Italiaander, R., and Brouwer, A. Presence of SARS-Coronavirus-2 RNA in Sewage and Correlation with Reported COVID19 Prevalence in the Early Stage of the Epidemic in the Netherlands. Environ. Sci. Technol. Lett. (2020) 7(7):511-516. doi:10.1021/acs.estlett.0c00357

Mehta, H. B., Ehrhardt, S., Moore, T. J., Segal, J. B., and Alexander, G. C. Characteristics of Registered Clinical Trials Assessing Treatments for COVID-19: a Cross-Sectional Analysis. BMJ Open (2020) 10(6):e039978. e039978. doi:10.1136/bmjopen-2020-039978

Menni, C., Valdes, A. M., Freidin, M. B., Sudre, C. H., Nguyen, L. H., Drew, D. A., et al. Real-time Tracking of Self-Reported Symptoms to Predict Potential COVID-19. Nat. Med. (2020) 26(7):1037-1040. doi:10.1038/s41591-0200916-2

Meoni, G., Ghini, V., Maggi, L., Vignoli, A., Mazzoni, A., Salvati, L., et al. Metabolomic/lipidomic Profiling of COVID-19 and Individual Response to
Tocilizumab. Plos Pathog. (2021) 17(2):e1009243. e1009243. doi:10.1371/ journal.ppat.1009243

Messner, C. B., Demichev, V., Bloomfield, N., Yu, J. S. L., White, M., Kreidl, M., et al. Ultra-fast Proteomics with Scanning SWATH. Nat. Biotechnol. (2021) 39(7):846-854. doi:10.1038/s41587-021-00860-4

Messner, C. B., Demichev, V., Wendisch, D., Michalick, L., White, M., Freiwald, A., et al. Ultra-high-throughput Clinical Proteomics Reveals Classifiers of COVID-19 Infection. Cel Syst. (2020) 11(1):11-24. doi:10. 1016/j.cels.2020.05.012

Morens, D. M., Breman, J. G., Calisher, C. H., Doherty, P. C., Hahn, B. H., Keusch, G. T., et al. The Origin of COVID-19 and Why it Matters. Am. J. Trop. Med. Hyg. (2020) 103(3):955-959. doi:10.4269/ajtmh.20-0849

Mou, L., Zhang, Y., Feng, Y., Hong, H., Xia, Y., and Jiang, X. Multiplexed Lab-OnA-Chip Bioassays for Testing Antibodies against SARS-CoV-2 and its Variants in Multiple Individuals. Anal. Chem. (2022) 94(5):2510-2516. Jan 26. doi:10. 1021/acs.analchem.1c04383

Munayco, C. V., Tariq, A., Rothenberg, R., Soto-Cabezas, G. G., Reyes, M. F., Valle, A., et al. Early Transmission Dynamics of COVID-19 in a Southern Hemisphere Setting: Lima-Peru: February 29th-March 30th, 2020. Infect. Dis. Model. (2020) 5(5):338-345. February 29th-March 30th. doi:10.1016/j. idm.2020.05.001

Murr, C., Grammer, T. B., Meinitzer, A., Kleber, M. E., März, W., and Fuchs, D. Immune Activation and Inflammation in Patients with Cardiovascular Disease Are Associated with Higher Phenylalanine to Tyrosine Ratios: the Ludwigshafen Risk and Cardiovascular Health Study. J. Amino Acids (2014) 2014:783730. doi:10.1155/2014/783730

Nachtigall, F. M., Pereira, A., Trofymchuk, O. S., and Santos, L. S. Detection of SARS-CoV-2 in Nasal Swabs Using MALDI-MS. Nat. Biotechnol. (2020) 38(10):1168-1173. doi:10.1038/s41587-020-0644-7

Nalbandian, A., Sehgal, K., Gupta, A., Madhavan, M. V., McGroder, C., Stevens, J. S., et al. Post-acute COVID-19 Syndrome. Nat. Med. (2020) 27(4):601-615. doi:10.1038/s41591-021-01283-z

Okolo, G. I., Katsigiannis, S., Althobaiti, T., and Ramzan, N. On the Use of Deep Learning for Imaging-Based COVID-19 Detection Using Chest X-Rays. Sensors (Basel) (2021) 21(17):5702. doi:10.3390/s21175702

Peacock, T. P., Penrice-Randal, R., Hiscox, J. A., and Barclay, W. S. SARS-CoV-2 One Year on: Evidence for Ongoing Viral Adaptation. J. Gen. Virol. (2021) 102(4):001584. doi:10.1099/jgv.0.001584

Platto, S., Xue, T., and Carafoli, E. COVID19: an Announced Pandemic. Cell Death Dis (2020) 11(9):799. doi:10.1038/s41419-020-02995-9

Renuse, S., Vanderboom, P. M., Maus, A. D., Kemp, J. V., Gurtner, K. M., Madugundu, A. K., et al. A Mass Spectrometry-Based Targeted Assay for Detection of SARS-CoV-2 Antigen from Clinical Specimens. EBioMedicine (2021) 69:103465. doi:10.1016/j.ebiom.2021.103465

Rijs, C., and Fenter, F. The Academic Response to COVID-19. Front. Public Health (2020) 8:621563. doi:10.3389/fpubh.2020.621563

Riva, L., Yuan, S., Yin, X., Martin-Sancho, L., Matsunaga, N., Pache, L., et al. Discovery of SARS-CoV-2 Antiviral Drugs through Large-Scale Compound Repurposing. Nature (2020) 586(7827):113-119. doi:10.1038/s41586-0202577-1

Ruszkiewicz, D. M., Sanders, D., O’Brien, R., Hempel, F., Reed, M. J., Riepe, A. C., et al. Diagnosis of COVID-19 by Analysis of Breath with Gas ChromatographyIon Mobility Spectrometry - a Feasibility Study. EClinicalMedicine (2020) 2930:100609. doi:10.1016/j.eclinm.2020.100609

Sanchez-Vazquez, R., Guío-Carrión, A., Zapatero-Gaviria, A., Martínez, P., and Blasco, M. A. Shorter Telomere Lengths in Patients with Severe COVID-19 Disease. Aging (2021) 13(1):1-15. doi:10.18632/aging.202463

Sauer, U., and Zamboni, N. From Biomarkers to Integrated Network Responses. Nat. Biotechnol. (2008) 26(10):1090-1092. doi:10.1038/nbt1008-1090

Shannon, A., Le, N. T.-T., Selisko, B., Eydoux, C., Alvarez, K., Guillemot, J.-C., et al. Remdesivir and SARS-CoV-2: Structural Requirements at Both Nsp12 RdRp and Nsp14 Exonuclease Active-Sites. Antiviral Res. (2020) 178:104793. doi:10. 1016/j.antiviral.2020.104793

Shen, B., Yi, X., Sun, Y., Bi, X., Du, J., Zhang, C., et al. Proteomic and Metabolomic Characterization of COVID-19 Patient Sera. Cell. (2020) 182(1):59-72. doi:10. 1016/j.cell.2020.05.032

Shi, Z., and Puyo, C. A. N-acetylcysteine to Combat COVID-19: an Evidence Review. Tcrm (2020) 16:1047-1055. doi:10.2147/TCRM.S273700 
Siemens Healthineers. Leveraging AI in the Prediction of COVID-19 Progression, Atellica COVID-19 Severity Algorithm (2021). Available at: https://www. siemens-healthineers.com/laboratory-diagnostics/atellica-portfolio/covid-19algorithm (Accessed November2021 25th.

SoRelle, J. A., Patel, K., Filkins, L., and Park, J. Y. Mass Spectrometry for COVID-19. Clin. Chem. (2020) 66(11):1367-1368. doi:10.1093/clinchem/ hvaa222

Spick, M., Lewis, H. M., Wilde, M. J., Hopley, C., Huggett, J., and Bailey, M. J. Systematic Review with Meta-Analysis of Diagnostic Test Accuracy for COVID-19 by Mass Spectrometry. Metabolism (2022) 126:154922. doi:10. 1016/j.metabol.2021.154922

Strick-Marchand, H., Morosan, S., Charneau, P., Kremsdorf, D., and Weiss, M. C. Bipotential Mouse Embryonic Liver Stem Cell Lines Contribute to Liver Regeneration and Differentiate as Bile Ducts and Hepatocytes. Proc. Natl. Acad. Sci. (2004) 101(22):8360-8365. doi:10.1073/pnas.0401092101

Struwe, W., Emmott, E., Bailey, M., Sharon, M., Sinz, A., Corrales, F. J., et al. The COVID-19 MS Coalition-Accelerating Diagnostics, Prognostics, and Treatment. The Lancet (2020) 395(10239):1761-1762. doi:10.1016/S01406736(20)31211-3

Subramanya, S. H., Czyż, D. M., Acharya, K. P., and Humphreys, H. The Potential Impact of the COVID-19 Pandemic on Antimicrobial Resistance and Antibiotic Stewardship. Virusdisease (2021) 32(2):1-8. doi:10.1007/ s13337-021-00695-2

Taquet, M., Dercon, Q., Luciano, S., Geddes, J. R., Husain, M., and Harrison, P. J. Incidence, Co-occurrence, and Evolution of Long-COVID Features: A 6-month Retrospective Cohort Study of 273,618 Survivors of COVID-19. Plos Med. (2021) 18(9):e1003773. e1003773. doi:10.1371/journal.pmed. 1003773

Thomson, E. C., Rosen, L. E., Shepherd, J. G., Spreafico, R., da Silva Filipe, A., Wojcechowskyj, J. A., et al. Circulating SARS-CoV-2 Spike N439K Variants Maintain Fitness while Evading Antibody-Mediated Immunity. Cell. (2021) 184(5):1171-1187. Wojcechowskyj JA, Davis C, Piccoli L, Pascall DJ, Dillen J, Lytras S. doi:10.1016/j.cell.2021.01.037

Tiwari, R., Dhama, K., Sharun, K., Iqbal Yatoo, M., Malik, Y. S., Singh, R., et al. COVID-19: Animals, Veterinary and Zoonotic Links. Vet. Q. (2020) 40(1): 169-182. doi:10.1080/01652176.2020.1766725

Tran, N. K., Howard, T., Walsh, R., Pepper, J., Loegering, J., Phinney, B., et al. Novel Application of Automated Machine Learning with MALDI-TOF-MS for Rapid High-Throughput Screening of COVID-19: a Proof of Concept. Sci. Rep. (2021) 11(1):8219. doi:10.1038/s41598-021-87463-w

Tse, L. V., Meganck, R. M., Graham, R. L., and Baric, R. S. The Current and Future State of Vaccines, Antivirals and Gene Therapies against Emerging Coronaviruses. Front. Microbiol. (2020) 11:658. doi:10.3389/fmicb.2020.00658

Turoňová, B., Sikora, M., Schürmann, C., Hagen, W. J., Welsch, S., Blanc, F. E., et al. In Situ structural Analysis of SARS-CoV-2 Spike Reveals Flexibility Mediated by Three Hinges. Science (2020) 370(6513):203-208. doi:10.1126/ science.abd5223

Van Doremalen, N., Bushmaker, T., Morris, D. H., Holbrook, M. G., Gamble, A., Williamson, B. N., et al. Aerosol and Surface Stability of SARS-CoV-2 as Compared with SARS-CoV-1. N. Engl. J. Med. (2020) 382(16):1564-1567. doi:10.1056/NEJMc2004973

Walsh, K. A., Jordan, K., Clyne, B., Rohde, D., Drummond, L., Byrne, P., et al. SARS-CoV-2 Detection, Viral Load and Infectivity over the Course of an Infection. J. Infect. (2020) 81(3):357-371. doi:10.1016/j.jinf.2020.06.067

Wang, L., Sievert, D., Clark, A. E., Lee, S., Federman, H., Gastfriend, B. D., et al. A Human Three-Dimensional Neural-Perivascular 'assembloid' Promotes Astrocytic Development and Enables Modeling of SARS-CoV-2 Neuropathology. Nat. Med. (2021) 27(9):1600-1606. doi:10.1038/s41591021-01443-1

Wang, P., Nair, M. S., Liu, L., Iketani, S., Luo, Y., Guo, Y., et al. Antibody Resistance of SARS-CoV-2 Variants B.1.351 and B.1.1.7. Nature (2021) 593(7857): 130-135. doi:10.1038/s41586-021-03398-2
Wang, Y., Zhang, D., Du, G., Du, R., Zhao, J., Jin, Y., et al. Remdesivir in Adults with Severe COVID-19: a Randomised, Double-Blind, Placebo-Controlled, Multicentre Trial. The Lancet (2020) 395(10236):1569-1578. doi:10.1016/ S0140-6736(20)31022-9

Ward, H., Cooke, G. S., Atchison, C., Whitaker, M., Elliott, J., Moshe, M., et al. Prevalence of Antibody Positivity to SARS-CoV-2 Following the First Peak of Infection in England: Serial Cross-Sectional Studies of 365,000 Adults. The Lancet Reg. Health - Europe (2021) 4:100098. doi:10.1016/j.lanepe.2021.100098

Wei, X., Zeng, W., Su, J., Wan, H., Yu, X., Cao, X., et al. Hypolipidemia Is Associated with the Severity of COVID-19. J. Clin. Lipidol. (2020) 14(3): 297-304. doi:10.1016/j.jacl.2020.04.008

Wierbowski, S. D., Liang, S., Liu, Y., Chen, Y., Gupta, S., Andre, N. M., et al. A 3D Structural SARS-CoV-2-Human Interactome to Explore Genetic and Drug Perturbations. Nat. Methods (2021) 18(12):1477-1488. doi:10.1038/s41592021-01318-w

Wiersinga, W. J., Rhodes, A., Cheng, A. C., Peacock, S. J., and Prescott, H. C. Pathophysiology, Transmission, Diagnosis, and Treatment of Coronavirus Disease 2019 (COVID-19). JAMA (2020) 324(8):782-793. doi:10.1001/jama. 2020.12839

Wise, J. Covid-19: Study Reveals Six Clusters of Symptoms that Could Be Used as a Clinical Prediction Tool. BMJ (2020) 370:m2911. doi:10.1136/bmj.m2911

World Health Organization. (2021). WHO Coronavirus (COVID-19) Dashboard. Available at: https://covid19.who.int [Accessed December 17 $7^{\text {th }}, 2021$ ].

Wu, D., Shu, T., Yang, X., Song, J.-X., Zhang, M., Yao, C., et al. Plasma Metabolomic and Lipidomic Alterations Associated with COVID-19. Natl. Sci. Rev. (2020) 7(7):1157-1168. doi:10.1093/nsr/nwaa086

Xie, Y., Xu, E., Bowe, B., and Al-Aly, Z. Long-Term Cardiovascular Outcomes of COVID-19. Nat Med. (2022). doi:10.1038/s41591-022-01689-3

Yu, M., Zhu, Y., Cong, Q., and Wu, C. Metabonomics Research Progress on Liver Diseases. Can. J. Gastroenterol. Hepatol. (2017) 2017:8467192. doi:10.1155/ 2017/8467192

Yuan, Z. C., and Hu, B. Mass Spectrometry-Based Human Breath Analysis: towards COVID-19 Diagnosis and Research. J. Anal. Test. (2021) 1-11. doi:10.1007/s41664-021-00194-9

Zhang, Y., Cai, X., Ge, W., Wang, D., Zhu, G., Qian, L., et al. Potential Use of Serum Proteomics for Monitoring COVID-19 Progression to Complement RT-PCR Detection. J. Proteome Res. (2021). acs.jproteome.1c00525. doi:10.1021/acs. jproteome.1c00525

Zhong, L., Zhu, L., and Cai, Z. W. Mass Spectrometry-Based Proteomics and Glycoproteomics in COVID-19 Biomarkers Identification. A. Mini-review. J. Anal. Test. (2021) 1-16. doi:10.1007/s41664-021-00197-6

Zhu, L., She, Z.-G., Cheng, X., Qin, J.-J., Zhang, X.-J., Cai, J., et al. Association of Blood Glucose Control and Outcomes in Patients with COVID-19 and Preexisting Type 2 Diabetes. Cel Metab. (2020) 31(6):1068-1077. doi:10.1016/j. cmet.2020.04.021

Conflict of Interest: The author declares that the research was conducted in the absence of any commercial or financial relationships that could be construed as a potential conflict of interest.

Publisher's Note: All claims expressed in this article are solely those of the authors and do not necessarily represent those of their affiliated organizations, or those of the publisher, the editors and the reviewers. Any product that may be evaluated in this article, or claim that may be made by its manufacturer, is not guaranteed or endorsed by the publisher.

Copyright (๑) 2022 Ibáñez. This is an open-access article distributed under the terms of the Creative Commons Attribution License (CC BY). The use, distribution or reproduction in other forums is permitted, provided the original author(s) and the copyright owner(s) are credited and that the original publication in this journal is cited, in accordance with accepted academic practice. No use, distribution or reproduction is permitted which does not comply with these terms. 